Chirurg 2016 $87: 1079$

DOI 10.1007/s00104-016-0322-y

Online publiziert: 4. November 2016

(c) Springer-Verlag Berlin Heidelberg 2016

CrossMark

F. Rauchfuß - U. Settmacher

Klinik für Allgemein-, Viszeral- und Gefäßchirurgie, Universitätsklinikum Jena, Jena, Deutschland

\title{
Stellenwert der Tumorgröße bei der Resektion hepatozellulärer Karzinome
}

war bei den großen Tumoren deutlich höher ( $82,6 \%$ vs. $31,7 \%$; $p<0,001)$.

Das Gesamtüberleben war zwischen beiden Gruppen vergleichbar (medianes Überleben 39 Monate [große HCCs] vs. 65 Monate [kleine HCCs], $p=0,213$ ). Prognosebestimmende Faktoren einer univariaten Analyse waren eine aufgetretene Tumorruptur, das Vorhandensein von Satellitenherden, eine mikrovaskuläre Infiltration, ein alpha-Fetoprotein (AFP) $>400 \mathrm{ng} / \mathrm{ml}$ sowie die perioperative Notwendigkeit von Transfusionen. In der multivariaten Analyse waren das Vorhandensein von Satellitenherden sowie die Transfusionsnotwendigkeit signifikante outcomebestimmende Faktoren.

Diskussion. Die hier vorgestellte Arbeit beschreibt eine Fallserie von Patienten mitgroßen hepatozellulären Karzinomen $(>10 \mathrm{~cm})$, die mittels Leberteilresektion therapiert wurden. Die Tumorgröße selbst war kein outcomebestimmender Faktor. Als prognosebestimmend wurden das Vorhandensein von Satellitenherden und damit eine fortgeschrittene Tumorerkrankung bzw. eine ungünstige Tumorbiologie sowie die Notwendigkeit von Bluttransfusionen detektiert. Bei letztgenannter Variable werden pathogenetisch immunmodulatorische Effekte vermutet.

Fazit. Die Größe eines hepatozellulären Karzinoms alleine sollte keine Kontraindikation zur Leberteilresektion darstellen. Auch große HCCskönnen mit akzeptablem Outcome operiert werden. Sollten allerdings bereits Satellitenherde vorhanden sein, muss die Indikation zur chirur- gischen Therapie sorgfältig abgewogen werden.

\section{Korrespondenzadresse}

PD Dr. F. Rauchfuß

Klinik für Allgemein-, Viszeral- und Gefäßchirurgie, Universitätsklinikum Jena Erlanger Allee 101, 07747 Jena, Deutschland falk.rauchfuss@med.uni-jena.de

Interessenkonflikt. F. Rauchfuß und U. Settmacher geben an, dass kein Interessenkonflikt besteht. 